\title{
Available supports and resources for postsecondary students with traumatic brain injury: A systematic review of the literature
}

\author{
Emily Tarconish*, Allison Lombardi, Joseph Madaus, Ashley Taconet and Carl Coelho \\ University of Connecticut, Storrs, CT, USA
}

Received 12 September 2020

Accepted 30 October 2020

\begin{abstract}
.
BACKGROUND: Postsecondary students with traumatic brain injuries (TBI) are a rapidly growing population, encompassing those who sustained injuries prior to attending postsecondary education and those who endure injuries during their postsecondary studies. Not only do these individuals face a broad range of symptoms, all of which can affect academic achievement, but they also do not achieve comparable academic outcomes to their peers without disabilities.

OBJECTIVE: There is a need to develop and examine the effectiveness of available supports and resources to meet the needs of these students.

METHODS: Twenty-three articles were systematically reviewed to illustrate what supports are currently described in the literature for postsecondary students with TBI and what research methods were used to assess the effectiveness of these supports.

RESULTS: Three categories of supports emerged, including concussion management protocols, typical study/learning strategies and accommodations, and interventions developed specifically for this population. Findings also indicated a lack of rigorous research methods used to assess these interventions' effects.

CONCLUSIONS: Implications for future research include a need for additional study of all supports and resources described in this review, and assessment of whether or not education professionals, including postsecondary disability services professionals, are aware of and using the tools and strategies addressed in this review.
\end{abstract}

Keywords: Traumatic brain injury, postsecondary education, supports, interventions, disability

\section{Introduction}

Hundreds of thousands of children and adolescents sustain a Traumatic Brain Injury (TBI) every year in

*Address for correspondence: Emily Tarconish, M.S., Department of Educational Psychology, University of Connecticut, Unit 3064 Charles B. Gentry Bldg 002, 249 Glenbrook Rd, Storrs, CT 06269, USA. Tel.: +1 570778 5019; E-mail: emily. tarconish@uconn.edu. the United States (CDC, 2017) and many of these young people go on to attend postsecondary education (LaForce \& Martin-McLeod, 2001; Krause \& Richards, 2014; Cahill et al., 2014). Recent statistics on postsecondary access are not available, but the National Longitudinal Transition Study-2 reported in 2011 that 8 years out of high school, $56 \%$ of high school exiters with TBI enrolled in some type of postsecondary education program (Newman et al., 2011). Individuals may also sustain TBI during their 
time in postsecondary education, such as collegiate athletes. Others, such as veterans, another group entering postsecondary education in high numbers, are also at increased risk to have experienced TBI. However, approximately one half of postsecondary students with TBI do not graduate within six years, and those who do, often complete fewer classes or achieve lower grades than their peers without disabilities (Newman et al., 2011; Todis et al., 2011). Further, most students with TBI do not disclose their injuries or seek support services, which may be related to these diminished outcomes. As incidence of TBI remain on the rise for students participating in postsecondary education, offices of disability services (ODS) need to find ways to help these individuals access services and develop programs that will meet their distinct needs. The professional literature cannot only serve as a guide for disability services professionals, but can also influence policy, and the training of future professionals (Mastropieri et al., 2009). This manuscript first presents an overview of the diagnostic criteria, symptomology, and incidence of TBI. Next, the growth of this population in postsecondary education environments and research describing the experiences of these students is outlined. Finally, the methods, results and conclusions of a systematic literature review synthesizing supports and resources for postsecondary students with TBI are discussed.

\subsection{Traumatic brain injuries: An overview}

A TBI encompasses an injury that causes a "disruption in the normal function of the brain that can be caused by a bump, blow, or jolt to the head, or penetrating injury," (CDC, 2019) caused by an external force (Blythe \& Bazarian, 2010). The Individuals with Disabilities Education Act (IDEA) defines TBI as "an acquired injury to the brain caused by an external physical force, resulting in total or partial functional disability or psychosocial impairment, or both, that adversely affects a child's educational performance. Traumatic brain injury applies to open or closed head injuries resulting in impairments in one or more areas, such as cognition; language; memory; attention; reasoning; abstract thinking; judgment; problem-solving; sensory, perceptual, and motor abilities; psychosocial behavior; physical functions; information processing; and speech. Traumatic brain injury does not apply to brain injuries that are congenital or degenerative, or to brain injuries induced by birth trauma" (IDEA, 1990).
TBI is classified into one of three severities: mild, moderate, or severe. The two factors that determine severity include duration of unconsciousness and post traumatic amnesia (PTA), or a disorder in episodic memory (Nakase-Richardson et al., 2011). A mild TBI, or concussion, involves 0-30 minutes of unconsciousness, and a period of 0-1 day of PTA; a moderate TBI entails between 30 minutes and 24 hours of unconsciousness and PTA of greater than one, but less than seven days, and a severe TBI includes loss of consciousness for over 24 hours and a period of PTA that is greater than 7 days. Approximately $75 \%$ of TBIs are considered mild and for approximately $15-30 \%$ of people with mild TBI, symptoms can be long-term or permanent, especially if individuals have endured multiple mild TBIs (Linquist et al., 2017).

\subsection{Symptoms of TBI}

TBIs can cause a range of impairments, including those affecting cognitive, physical, emotional, behavioral, psychological, and social abilities (Mealings et al., 2012). Cognitive impediments can affect short-term and working memory, concentration, processing speed, cognitive flexibility, maintaining newly acquired skills, and generalizing information to new situations. Executive functioning abilities can be impaired, including organizational, decision making, planning, and initiation abilities. Cognitive deficits can also be exacerbated by physical symptoms, such as fatigue, headaches, or dizziness (Hux et al., 2010).

Psychological symptoms, including anger, depression, mood swings, anxiety, feeling overwhelmed, and irritability, as well as behavioral changes, such as impulsivity, inflexibility, low-frustration tolerance, ego-centrism, low self-efficacy, lack of initiative, and black and white thinking patterns can complicate cognitive processes as well as disrupt a survivor's ability to seek resources or support. Further, as individuals deal with TBI symptoms, they may experience social withdraw or isolation, and difficulty relearning social cues or maintaining relationships (Hux et al., 2010; Kennedy et al., 2008a; Stewart-Scott \& Douglas, 1998).

In addition to the range of impairments that may occur after injury, survivors may not even recognize some of these changes due to diminished, or compromised, self-awareness. Survivors of TBI may develop inaccurate perceptions of their strengths and weaknesses, and have trouble realizing pre-post injury differences, which can lead to unrealistic goal setting, 
and a refusal to accept post-injury abilities, or to seek help (Bush et al., 2011; Childers \& Hux, 2016; Kennedy et al., 2008a; Martinez \& Davalos, 2016).

A further complication involves day-to-day variation in symptoms and unpredictable periods of improvement (Bush et al., 2011). These changes can be typical as the brain heals; however, they can confuse or complicate a survivor's ability to understand progress and seek supports (Daneshvar et al., 2011). Additional factors, such as age of injury, severity of injury, presence of comorbid conditions, and quantity and quality of rehabilitation received, can all influence the symptoms that a survivor of TBI experiences.

\subsection{A growing and diverse population in postsecondary education}

Postsecondary students who experience TBI encompass a diverse group, including those who sustained injuries in childhood or adolescence prior to attending postsecondary education, those who endured injuries while attending higher education, and adults who experience injury and enroll in higher education postinjury to pursue alternative careers. Regarding the first of these groups, TBI is one of the leading causes of acquired disability for children and adolescents. Affecting approximately 329,290 young people each year, individuals ages $0-4$ and 15-19 are two of the most likely groups to experience this disability. The Center for Disease Control (CDC) also suggests that this number underestimates the actual prevalence, as many people do not seek treatment for mild TBI (CDC, 2017). With nearly $70 \%$ of high school graduates enrolling in postsecondary education (Bureau of Labor Statistics, 2019), one can assume that many students with TBI are included in this population. And as previously noted, $56 \%$ of high school exiters with TBI enroll in some type of postsecondary education (Newman et al., 2011).

Typical college-aged students, ages 19-24, are another of the most likely age-groups to endure TBI (CDC, 2017), and specific groups, such as student athletes, face increased risks. Previous estimates from the CDC (2017) suggest that 300,000 sports-related TBIs occur each year; however, this calculation only considers athletes who lost consciousness as a result of injury. This, coupled with the lack of athletes reporting injury, leads some researchers to estimate that 1.6 to 3.8 million sports related TBIs actually occur every year (Daneshvar et al., 2011).
Veterans are also more likely to experience TBI. Currently, the largest influx of veterans is enrolling in postsecondary education since World War II (Sinski, 2012). It is estimated that between 11-23\% of servicemen and women currently deployed and $19 \%$ of veterans experience TBI (Linquist et al., 2017; Madaus et al., 2009). An additional complication affecting veterans is the prevalence of comorbid conditions, including PTSD, which can exacerbate TBI symptoms. These individuals are also less likely to self-disclose and seek supports for their disabilities than non-veteran students with disabilities (Church, 2009; Shackelford, 2009).

Another issue affecting postsecondary students with TBI includes adapting to acquired injury. Those who acquire TBI in adolescence or young adulthood may not have had time to fully understand their changed abilities and needs; they may also not be familiar with services or accommodations or how to obtain them. Receiving postsecondary disability supports requires students to self-disclose their disabilities as well as self-advocate to obtain needed resources (Madaus, 2010). People with newly acquired disabilities, such as TBI, may not have exercised these abilities and may be less likely to disclose or seek supports (Bush et al., 2011). Additionally, after TBI, individuals' interests, preferences and abilities may be altered; however, survivors may still feel pressure to pursue pre-injury plans or face expectations from others based on their pre-injured abilities (Todis \& Glang, 2008; MacLennan \& MacLennan, 2008).

Postsecondary students with TBI remain a unique population to accommodate and support and this group only continues to grow. Researchers have surveyed groups of undergraduate students and found between $10 \%$ to 39\% endorsed criteria for TBI (La Force \& Martin-McLeod, 2001; Krause \& Richards, 2014; Cahill et al., 2014). While these students may experience symptoms that can occur after TBI for other reasons, such as identifying with a different disability, this finding may also indicate the growing prevalence of TBI in college students. Facing the increased need to accommodate this population, researchers have begun to examine these students' experiences.

\subsection{Studies examining experiences of postsecondary students with TBI}

Studies have emerged examining the descriptive experiences of postsecondary students with TBI. This 
line of research has revealed that the effects of TBI symptoms may be amplified in postsecondary education environments, which present increased cognitive and academic demands, as well as opportunities for social development. TBI cognitive sequelae can complicate every aspect of learning, including paying attention to information, processing, and retrieving what one has learned, and can be further exacerbated by physical impediments. Overall, symptoms led students with TBI to expend much more effort to learn than prior to their injuries. However, despite doing more, these students also reported lower productivity and grades and reduced satisfaction with postsecondary education (Hux et al., 2010; Kennedy et al., 2008a; Stewart-Scott \& Douglas, 1998).

Students also reported emotional and psychosocial difficulties relating to both challenges produced by the injury itself, as well as to difficulties adjusting to the consequences of recovery, such as feelings of loss (Hux et al., 2010), and reduced self-confidence and self-esteem (Stewart-Scott \& Douglas, 1998). Additionally, as students spent more time and energy on learning tasks, they had less time to participate in extra-curricular activities and noted changes to their social relationships (Hux et al., 2010; Stewart-Scott \& Douglas, 1998).

While several studies have captured the experiences of postsecondary students with TBI, few have examined the resources and services that are effective for this population. This systematic literature review addressed the following research questions:

1) What academic supports are described in the literature for postsecondary students with TBI?

2) What methods are used to evaluate the effectiveness of these supports?

\section{Method}

A search of four electronic databases was conducted, including ERIC, PSYCHINFO, Academic Search Premier, and Psychological and Behavioral Sciences, to identify articles for review. Initial search terms included ("traumatic brain injur*" OR "head injur*” OR "brain injur*” OR "TBI" OR "traumatic acquired brain injur*" OR "brain trauma*" OR "concussion*" OR "acquired brain injur*") AND ("postsecondary education" OR "post secondary education" OR "higher education" OR "university" OR "college" OR "community college" OR "undergraduate education" OR "college athletes"). This combination identified 63,017 articles. To further narrow the search, AND ("student*") was added to the search, which resulted in 6217 articles. A fourth search term, AND ("accommodation"” OR "learning strateg*" OR "management" OR "intervention*" OR "education") was added, and 1624 articles were identified. Examination of articles published after 1990 that were written in English reduced the number of articles to 1568 . Additionally, as protections for postsecondary students with disabilities vary by country (United Nations, 2019), this review will focus on articles published in the United States. The date range, 1990-2019, was selected as it corresponded to the period after which the Americans with Disabilities Act (ADA) began, which is one of the principle laws governing provision of accommodations to students with disabilities in higher education. While Section 504 of the Rehabilitation Act (1973) also provided protections for students with disabilities in postsecondary education, TBI was not added to the Individuals with Disabilities Education Act (IDEA) until 1990, and thus, may have not been a frequently considered disability in education settings until this time. As IDEA coincided with the inception of the ADA, the date range was selected to begin in 1990 . After duplicates were removed, the search produced 1206 unique articles for abstract screening.

\subsection{Inclusion criteria}

The literature on students with TBI reveals a lack of consistent language among both researchers and practitioners used to describe this injury (Weber \& Edwards, 2010). In order to capture relevant literature using a variety of terminology, the search string included a range of terms, such as traumatic brain injur*, head injur*, brain injur*, TBI, traumatic acquired brain injur*, brain trauma*, concussion*, acquired brain injury. The review included articles that focused on students with both acquired and traumatic brain injuries as a primary diagnosis. For the purpose of this literature review, "postsecondary education," was defined as education experiences that take place after the completion of (K-12) schooling, and lead to completion of a training certificate or diploma. Articles that described supports that were used in both K-12 and postsecondary settings were also included, but only the data relating to postsecondary students was coded in these articles. The third search term, "student," was added to focus the search on articles that discussed studies that focused on those learning in postsecondary settings, as opposed to research that was conducted in these 
settings and not necessary for students. The final search string, "accommodation*" OR "management" OR "intervention*" OR "learning strateg*" OR "education" was used to narrow the search to focus on academic interventions or supports that postsecondary students with TBI use or receive. The first two terms were selected as they reflect the language used to describe formal academic support services provided to students with disabilities in postsecondary education. The next three terms were used to capture learning tools or methods that students with TBI can use to independently accommodate themselves. As few resources for postsecondary students with TBI have been empirically assessed, this review was scoping in its purview, and included non-data-based articles in order to capture all resources described in the literature. In order to be considered data-based, articles had to describe data-collection or analysis, and include methods and results. Non-data-based studies included descriptions of programs that did not include data-collection or analysis.

\subsection{Exclusion criteria}

In conjunction with the above inclusion criteria, articles that did not solely focus on students with TBIs were excluded from the review. Additionally, to focus on articles examining postsecondary students with TBI, those that described elementary or secondary education settings, transition to postsecondary environments, or community or rehabilitation-based programs were not considered for review. The focus on interventions for students also led to the removal of articles about interventions conducted in postsecondary settings, but not for students themselves. The types of articles that were removed based on these criteria include the refinement of instruments to diagnosis TBI sequelae, the biochemical consequences of TBI, or medical interventions for TBI. Finally, articles that only focused on the experiences, such as the manifestation of symptoms, TBI prevention strategies, strategies for teaching students with TBI, or predictors of TBI were excluded from this search. Incorporating these exclusions, abstract screening removed the following articles, including those not about students with TBI (785), postsecondary students with TBI (275), or those that did not focus on accommodations, programs, interventions, or strategies for students with TBI (104). Of the remaining 42 articles, 19 were not peer-reviewed and were removed, resulting in 23 articles to be considered for full review.
Each of the 23 articles was coded according to the following criteria: school type, including community college, 2-year college, 4-year college, or university. It was also noted if the study also included $\mathrm{K}-12$ students in addition to postsecondary students; if the article presented original data; methods, including quantitative, qualitative, mixed methods, single subject, or none. Articles coded "none" included nondata-based articles, or those that described programs or interventions, but did not evaluate the resources' effectiveness. Finally, the type of resource or intervention described in each study was identified. The first author and an additional author coded all 23 of the articles on the criteria stated above. Coding was compared and the interrater reliability was calculated at $87.7 \%$. Discrepancies between coders were discussed, when needed, and consensus was reached.

\section{Results}

The earliest article retained provided an overview of services available to postsecondary students with TBI in 1991 and offered a historical perspective from which to frame the types of supports that are currently provided (Cook, 1991). Written immediately after the inception of the ADA, this article captured a time when not all colleges provided a range of services, and specifically lacked an individualized approach to accommodation provision. Depending on the size of the institution, the number of students with disabilities and other factors, resources were available to varying extents.

The remainder of articles that met the criteria for review reflected the adoption of current postsecondary disability services practices and fell into one of three: concussion management, learning strategies or accommodations, and interventions. A definition of each category and its description in the literature follows.

\subsection{Concussion management}

The review identified seven articles about concussion management, or systematic procedures to assist in the recovery and accommodation of students with mild TBI, typically for athletes in postsecondary environments. Although student athletes may encompass a small portion of undergraduate students, concussion management for these individuals was a prominent topic in the literature and was not described for any other group. These articles are included in the 
current review as they present procedures and protocols that may be useful for other postsecondary students with concussions. While the National Collegiate Athletic Association (NCAA) mandates that all college athletic programs must maintain concussion management protocols, the protocol requirements primarily relate to return to play; less guidance is provided regarding returning to academic participation or accommodating students who experience longterm symptoms (Baugh, et al., 2014; Aukerman et al., 2016; Kerr et al., 2015; Sohlberg \& Ledbetter, 2016).

The NCAA recommends that institutions devise and implement "Return to Learn" (RTL) protocols, but specific guidelines or requirements are not provided (Aukerman et al., 2016). The guidelines also recommend that student athletes who experience prolonged symptoms, for a period of over two weeks, should seek neuropsychological evaluation and consult with academic and disability professionals at the institution to determine if academic accommodations are needed (NCAA Sport Science Institute, 2017). One reason that NCAA RTL guidelines may be unspecific and not mandatory includes that evidence-based practices regarding RTL have not been established and they differ from institution to institution (Williamson et al., 2018). Most athletic trainers in both secondary and postsecondary education indicated that academic accommodations were included in the concussion management plans. Details about types or effectiveness of accommodations were not included (Williamson et al., 2018).

Hall et al. (2015) provided recommendations to concussion management teams and stressed the importance of students with TBI practicing cognitive rest before resuming academic work. Once students gradually resume cognitive tasks, Hall et al. (2015) suggested academic accommodations to alleviate symptoms, such as taking breaks while studying or in class, providing class materials, including notes, to students and in different formats, allowing students to work in smaller groups, to use extended time for exams or assignments, or to reschedule courses at a later time during the day. While providing cognitive rest to individuals who experience concussion is an evidenced-based practice, the effectiveness of proposed accommodations, including those mentioned by Hall et al. (2015), was not addressed.

Another issue included the inconsistency among institutions regarding who coordinates academic accommodations for students with concussions. Chinn and Porter (2013) found that a majority of athletic trainers indicated that they communicated directly with instructors to coordinate academic accommodations for student-athletes. In contrast, Knollman-Porter et al. (2014) found that it was the role of concussion clinic speech language pathologists to coordinate and notify professors of accommodations. Since policies related to coordinating concussion management may differ by institution, it may not be clear to students to whom they should report if they sustain a concussion, potentially increasing the likelihood that they do not seek supports.

\subsection{Learning strategies \& typical accommodations}

Three articles were identified that presented descriptions of general learning strategies or tools that are commonly used by postsecondary students with TBI; this category includes strategies and tools that were not specifically developed for students with TBI and may be used by any student. Brown et al. (2017) examined students' preferences regarding cognitive support technology. Students in this study preferred external, high-tech items, such as digital calendars, voice memos, electronic lists, scheduling apps, and "pop-up" reminders as tools that most closely met their cognitive needs. Effectiveness of preferred tools was not evaluated, but it was noted that for external aids to be most beneficial, students must be appropriately matched to technology, considering their needs, strengths, preferences, and previous experiences with technology (Scherer, 2005). Users should also receive training for and practice using the technology and be provided opportunities to provide feedback to experience optimal benefit from the tools (Brown et al., 2017).

Hux et al. (2010) identified learning strategies and accommodations used by postsecondary students with TBI. The researchers developed five sub-themes to encompass the supports that students used, including "study skills, relying on others, perseverance and attitude, self-advocacy, and seeking advice," (p. 22). Typical accommodations included receiving class notes or outlines ahead of time, working with study groups, using note takers, extended time on exams, homework, and in-class assignments, a scribe or computer for essay exams, course substitutions, a reduced course load, short-travel distance between classes, priority registration, a calculator for math assignments and exams, separate testing room for exams, and registering for small classes. "Relying on others" 
involved working with tutors in specific content areas, disability services professionals to develop study skills, and professors to clarify what to study. Students also noted that developing perseverance and self-advocacy skills helped them academically (Hux et al., 2010). A second study by Bush et al. (2011) suggested that limited self-awareness for students with TBI was likely to "prohibit consistent implementation of strategies to compensate for areas of weaknesses," or limit students' abilities to generalize these approaches (p. 245).

\subsection{Interventions}

Twelve articles described interventions that were used by postsecondary students with TBI and specifically developed for this population. For the purpose of this review, interventions are defined as specific forms of assistive technology, direct assistance, or strategy instruction; a literature review that addressed interventions for postsecondary students with learning disabilities used a similar definition (Zeng et al., 2018). One article summarized a comprehensive approach to working with students with TBI. Four articles described coaching, or instruction, strategies, including two for essay writing, one for reading comprehension, and one to improve metacognition and study skills. One article described a peer support group. The remaining six articles described a specific intervention, Project Career, involving cognitive support technology and intensive case management. The number of concussion management, learning strategies \& accommodations, and intervention articles, and descriptions of the intervention types can be found in Table 1.

\subsubsection{A comprehensive approach}

Sohlberg \& Ledbetter (2016) reviewed 24 cases of secondary and postsecondary students who experienced concussions and the services that they received

Table 1

Number of Studies and Type of Resource Described

\begin{tabular}{lc}
\hline Characteristic & Total \\
\hline Concussion management protocol & 7 \\
Study skills \& accommodations & 4 \\
Interventions & \\
$\quad$ Comprehensive approach & 1 \\
$\quad$ Cognitive support technology \& & 6 \\
$\quad$ intensive case management & \\
Metacognitive instruction & 1 \\
Writing intervention & 2 \\
Reading comprehension intervention & 1 \\
Peer support group & 1 \\
\hline
\end{tabular}

within a speech language clinic. The researchers described restorative and compensatory supports provided to students with TBI, classifying them into four categories: psychoeducational supports, direct attention training (DAT), metacognitive strategy instruction (MSI), assistive technology for cognition and psychoeducational supports. The most frequently provided resource $(n=24)$, psychoeducational supports, were intended to foster client's self-awareness and acceptance for the need for assistance. Thirteen students were provided DAT, a process that combines intensive exercises with metacognitive strategies to restore or improve impaired cognition functions. MSI was the next most common support $(n=9)$, which involves "systematic, step-by-step instruction in the use of strategies to facilitate selfregulation of states of mind, task preparation, and task execution," (Sohlberg \& Ledbetter, 2016, p. 143). The last approach described included training students to select and appropriately use assistive technology for cognition (ATC) to compensate for cognitive impairments; five students received this training, which included "planner books, calendars, and wristwatches, to specialized devices that use computer software and networking capabilities," and "e-readers, homework-tracking apps, smart pens for classroom note taking, and electronic calendars" (Sohlberg \& Ledbetter, 2016, p. 144). These four types of supports were delivered by speech language pathologists and graduate students.

Twenty-two of the 24 students discussed in the cases met their self-selected goals after receiving one or more of the four services for an average of once per week for an average of nine weeks. Goals related to academic outcomes, such as improved grades, increased number of completed assignments, and improved class attendance. Goals also pertained to cognitive functioning, such as decreased time to complete tasks, increased use of strategies, improvements in reading comprehension or sustained attention, and decreased symptom severity. Some students also achieved improvements in social skills (Sohlberg \& Ledbetter, 2016).

\subsubsection{Essay writing interventions}

Explicit instruction or feedback has been documented as an effective teaching strategy for students with TBI (Kennedy et al., 2008b). Dinnes \& Hux (2018) developed a multicomponent writing intervention using this approach. The intervention provided opportunities for the participant to practice 
semantic associations, semantic mapping, proofreading, written organization, foundational skills, and developing writing goals. The participant made gains in the quality of writing he produced, including decreases in redundancies, increases in the number of writing strategies used and percentage of correct writing sequences, as well as improvements in expanding semantic maps. However, he did not experience improvements relating to revision.

Ledbetter et al. (2019) assessed a writing intervention for postsecondary students with TBI that used computer prompts to help students self-monitor and regulate while writing. The researchers found a moderate functional relation between the computerbased prompting and improvements in overall writing quality scores, which included ideas, content, and organization. While the changes noted for organization were statistically significant, those relating to ideas and content were not. No functional relationship appeared between the intervention and revision abilities, voice, word choice, or sentence fluency.

\subsubsection{Reading comprehension intervention}

Incorporating metacognitive strategy instruction and computer-based prompts, Griffiths et al. (2016) devised a multi-component reading comprehension strategy package. This intervention intended to improve reading comprehension by providing prompts to support related functions, including attention working memory, and executive processes. Participants were able to recall more correct information units from the text in both immediate and delayed free recall tasks, as well as demonstrated improved local and global coherence of the reading.

\subsubsection{Metacognitive and general study skills training}

Kennedy \& Krause (2011) studied the effects of self-regulated learning coaching provided to two postsecondary students with TBI. After one academic year of using this coaching, the two student participants used more study and learning strategies, experienced functional gains in writing, and demonstrated some evidence of strategy generalization. One participant also spent less time studying as he used learning strategies more efficiently, while the other was able to develop more realistic goals and assess his own abilities with increased accuracy. Both participants also utilized formal academic accommodations more regularly.

\subsubsection{Peer support group}

Peer supports for postsecondary students with disabilities comprise a cost-effective and naturalistic way to offer support to students (Bialka et al., 2017). Chinn (2009) described an Acquired Brain Injury Students (ABIS) Club for students at a junior college. The group invited students to attend regular meetings, which provided support, and opportunities to learn about cognitive strategies and self-determination. It also offered opportunities to practice these and other skills, including leadership, social, problem solving, and decision making. While not an experimental study, the authors noted that students who participated in this group reported improved confidence, awareness of their abilities, and academic persistence.

\subsubsection{Project career}

Rumrill and colleagues (2016) developed an intervention, called "Project Career," that combined cognitive support technology with intensive case management services to improve career readiness and employment outcomes for postsecondary students with TBI. Cognitive support technology primarily included "apps" that helped students to compensate for TBI-related impairments or to develop additional learning or coping skills (Jacobs et al., 2017). A majority of participants in Project Career experienced a range of benefits, including increased rates of college retention and accommodation or support service use, participation in career preparatory activities, improvements in grades, career self-efficacy, quality of life, well-being, and levels of comfort using technology (Leopold et al., 2019). Participants also felt more positive toward academics and technology and expressed the ability to be more independent and social (Hendricks et al., 2015; Nardone et al., 2015).

\subsection{Research design}

The second research question identified the types of methods that were used to measure or evaluate

Table 2

Number of Articles by Methodology

\begin{tabular}{lc}
\hline Characteristic & Total \\
\hline Presented original data & 19 \\
Descriptive & \\
Comparative & 5 \\
Mixed methods & 6 \\
Qualitative & 1 \\
Single subject & 2 \\
Case study/review & 5 \\
Did not present original data & 4 \\
\hline
\end{tabular}


available resources and services for postsecondary students with TBI, as well as the characteristics of each study. This review characterized 19 studies (all based in the United States), eight of which took place in 4-year colleges or university settings, one in a 2-year college setting, six that addressed both 2 and 4-year settings, and one that discussed general postsecondary environments. Two additional articles described both $\mathrm{K}-12$ and postsecondary settings, although the review only incorporated the latter results. Most studies $(n=16)$ included students as participants, while three others assessed the perspective of the service providers, such as athletic trainers, clinicians, and coaches. Most sample sizes were below 150, although three larger studies included 327, 2,880 , and 4,000 participants. The most frequently discussed demographic characteristics were gender and age, while four studies also included race, and four others included no demographic information. Nine studies did not specifically clarify participants' brain injury severity; seven of these articles described concussion protocols, therefore, it can be assumed that these studies applied mostly to students with mild TBI. Three studies focused on students with mild $\mathrm{TBI} /$ concussion, one on those with mild to moderate TBI, two on those who experienced mild and severe

Table 3

Characteristics of Data-Based Studies

\begin{tabular}{lc}
\hline Characteristic & Total \\
\hline Setting & \\
4-year & 8 \\
2-year & 1 \\
2-year \& 4-year & 6 \\
K-12 \& postsecondary education & 2 \\
General postsecondary education & 1 \\
Population & \\
Students & 16 \\
Professionals & 3 \\
Sample size & \\
1-5 & 9 \\
6-25 & 3 \\
26-100 & 2 \\
100-150 & 2 \\
327+ & 3 \\
Demographics & \\
Sex \& age & 11 \\
Sex, age, \& race & 3 \\
Race \& age & 1 \\
Not specified & 4 \\
TBI severity & \\
Mild/concussion & 3 \\
Mild-moderate & 1 \\
Mild \& severe & 2 \\
Severe & 3 \\
Mild, moderate \& severe & 1 \\
Not specified & 9 \\
\hline
\end{tabular}

TBI, three on those with severe TBI, and one on those with mild, moderate, or severe TBI. A summary of the number of articles using each methodology and the characteristics of data-based studies can be found in Tables 2 and 3.

\section{Discussion}

This systematic literature review focused on two research questions. The first asked: What supports and resources are described in the literature for postsecondary students with traumatic brain injury (TBI)? Three categories of supports and resources for postsecondary students with TBI emerged from the literature: concussion management, study/learning strategies and accommodations, and interventions. Regarding concussion management, especially RTL protocols, this review found that some institutions do not maintain consistent policies, while others may have policies that remain unenforced. Further, no evidence-based practices exist regarding effective RTL protocols. These findings suggest that collegiate athletes who endure TBI may not all receive similar access to treatment and accommodation.

The articles focused on learning strategies and accommodations, as well as interventions, illustrate how and in what ways typical academic supports can be used to compensate for TBI-related impairments. Many interventions that were developed specifically for students with TBI focused on fostering metacognitive skills to help students re-evaluate their changed abilities and needs after injury. In contrast to a purely compensatory approach, which may be used by most postsecondary students with disabilities, interventions for students with TBI incorporated components of cognitive rehabilitation, focusing on both compensating for and restoring cognitive processes. This finding supports the need to ensure that accommodations that achieve both are present in postsecondary disability services settings. Further, this review identified only seven unique interventions for postsecondary students with TBI. There is not only a need to continue the creation and refinement of tools to meet the unique needs of this population, but also to assess the effects of these supports using rigorous research methods.

The second research question considered in this review sought to determine the types of methods used to evaluate the effectiveness of the supports and resources described. The 19 data-based articles presented case studies or reviews, comparative 
descriptive, qualitative, or mixed methods analyses. In addition to lacking rigorous, experimental methods to assess the effectiveness of the supports and resources, most of the studies that examined students had small sample sizes and thus limited generalizability. Studies also collected an inconsistent range of demographic characteristics, possibly further limiting generalizability. An omission of particular relevance includes the lack of TBI severity participants experienced. There is a need to express for individuals with different severities of TBI, as well as resulting level of cognitive impairments, what interventions are practical or efficacious. Future research should clarify if interventions function in similar ways for students with different injury severities and levels of cognitive impairment, or if interventions need to be modified based on these factors, and if so, how. Additionally, as the literature research suggests that students with TBI are not likely to use services or accommodations, researchers should consider assessing intervention effects on measures of academic performance, such as grades, GPA, classes passed/failed, and assignments completed, as well as the frequency with which students with TBI use the interventions.

\subsection{Implications for practice}

While interventions for postsecondary students with TBI are beginning to emerge, this literature review demonstrated that their implementation may be inconsistent or non-existent in some postsecondary settings. Areas of consideration for practitioners may include establishing clear and consistent procedures for college students who endure concussions to request and receive academic and other accommodations. Further, as only one article in this review mentioned restorative methods, it can be assumed that they are not widely used in postsecondary disability services. There may be a need for disability services professionals to incorporate restorative methods into the services they provide. Additionally, as vocational rehabilitation counselors may provide services to consumers with TBI who are enrolled in postsecondary education (State Vocational Rehabilitation Services Program, 2020), there is a need for these professionals to be familiar with relevant supports these students may use. As many of the articles describing TBI-specific interventions were found in journals targeting audiences in speech language pathology or athletic training fields, it is unclear if these practices are being used by educational practitioners. Future research is required to answer this question.

\subsection{Implications for research}

As most of the studies considered did not use experimental methods, nor large samples, there is a need to use rigorous methods and larger participant pools to evaluate the effectiveness of these interventions. Additionally, future studies should examine and include comprehensive demographic information in their analyses, including participant sex/gender, age, race and TBI severity. This information may provide a clearer picture of for whom the interventions are effective; if discrepancies are noted, future research can explore how interventions can potentially be modified to serve a broader population of students with TBI. Continued research using rigorous methods of analyses is necessary to evaluate the effectiveness of concussion management protocols, especially aspects regarding return to learn, as well as interventions that were developed to accommodate the specific needs of postsecondary students with TBI.

\section{Conflict of interest}

None to report.

\section{References}

Aukerman, D. F., Philips, N. R., \& Graham, C. (2016). Concussion management in the collegiate athlete. Sports Medicine and Arthroscopy Review, 24(3), 130-133. https://doi.org/ 10.1097/JSA.0000000000000118

Baugh, C. M., Kroshus, E., Daeshvar, D. H., Filali, N. A., Hiscox, M. J., \& Glantz, L. H. (2014). Concussion management in United States college sports. The American Journal of Sports Medicine, 43(1), 47-56. https://doi.org/10.1177/ 0363546514553090

Bialka, C. S., Morro, D., Brown, K., \& Hannah, G. (2017). Breaking barriers and building bridges: Understanding how a student organization attends to the social integration of college students with disabilities. Journal of Postsecondary Education \& Disability, 30(2), 157-172.

Blyth, B. J., \& Bazarian, J. (2010). Traumatic alterations in consciousness: Traumatic brain injury. Emergency Medical Clinics of North America, 28(3), 571-594. https:// doi.org/10.1016/j.emc.2010.03.003

Brown, J., Hux, K., Hey, M. \& Murphy, M. (2017). Exploring cognitive support use and preference by college students with TBI: A mixed-methods study. NeuroRehabilitation, 41, 483499. https://doi.org/10.3233/NRE-162065 
Bureau of Labor Statistics. (2019). College enrollment and work activity of recent high school and college graduates - 2018. Retrieved from https://www.bls.gov/news. release/hsgec.nr0.htm

Bush, E., Hux, K., Zickenfoose, S., Simanek, G., Holmberg, M., \& Ambyr, H. (2011). Learning and study strategies of students with traumatic brain injury: A mixed methods study. Journal of Postsecondary Education and Disability, 24(3), 231-250.

Cahill, S. M., Rotter, J. M., Lyons, K. K., \& Marrone, A. R. (2014). Survivors of brain injury: The narrative experiences of being a college or university student. Canadian Journal of Occupational Therapy, 81(2), 93-101. https://doi.org/10. 1177/000841741453318

Centers for Disease Control and Prevention (CDC). (2017). Rates of TBI-related emergency department visits by age groupUnited States, 2001-2010. Retrieved from https://www.cdc. gov/traumaticbraininjury/data/rates_ed_byage.html

Centers for Disease Control and Prevention (CDC). (2019). Traumatic Brain Injury \& Concussion. Retrieved from https:// www.cdc.gov/traumaticbraininjury/index.html

Childers, C., \& Hux, K. (2016). Invisible Injuries: The experiences of college students with histories of mild traumatic brain injury. Journal of Postsecondary Education and Disability, 29(4), 389- 405.

Chinn, N. R. (2009). Acquired brain injury club at a community college: Opportunities for support, involvement, and leadership. Journal of Postsecondary Education and Disability, 21(3), 175-178.

Chinn, N. R., \& Porter, P. (2013). Concussion management in community college athletics: Revealing and understanding the gap between knowledge and practice. Community College Journal of Research and Practice, 37(6), 409-423. https://doi. org/10.1080/10668926.2012.710127

Church, T. E. (2009). Returning veterans on campus with war related injuries and the long road back home. Journal of Postsecondary Education and Disability, 22(1) 43-52.

Cook, J. (1991). Higher education: An attainable goal for students who have sustained head injuries. Journal of Head Trauma Rehabilitation, 6(1), 64-72. https://doi.org/10.1097/ 00001199-199103000-00011

Daneshvar, D., Riley, D., Nowinski, C. (2011). Long-term consequences: effects on normal development profile after concussion. Physical Medicine and Rehabilitation Clinics of North America, 22, 683-700. https://doi.org/10.1016/j.pmr. 2011.08.009

Dinnes, C., \& Hux, K. (2018). A multicomponent writing intervention for a college student with mild brain injury. Communication Disorders Quarterly, 39(4), 490-500. https:// doi.org/10.1177/1525740117716416

Griffiths, G. G., Sohlberg, M. M., Kirk, C., Fickas, S., \& Biancarosa, G. (2016). Evaluation of use of reading comprehension strategies to improve reading comprehension of adult college students with acquired brain injury. Neuropsychological Rehabilitation, 26, (2), 161-190. https://doi.org/10. 1080/09602011.2015.1007878

Hall, E. E., Ketcham, C. J., Crenshaw, C. R., Baker, M. H., McConnell, J. M., \& Patel, K. (2015). Concussion management in collegiate student-athletes: Return-to-academics recommendations. Clinical Journal of Sports Medicine, 25(3), 291-296. https://doi.org/10.1097/JSM.0000000000000133

Hendricks, D. J., Sampson, E., Rumrill, P., Leopold, A., Elias, E., Jacobs, K., Nardone, A., Scherer, M., \& Stauffer, C.
(2015). Activities and interim outcomes of a multi-site development project to promote cognitive support technology use and employment success among postsecondary students with traumatic brain injuries. NeuroRehabilitation, 37, 449-458. https://doi.org/10.3233/NRE-151273

Hux, K., Bush, E., Zickenfoose, S., Holmberg, M., Henderson, A., \& Simanek, G. (2010). Exploring the study skills and accommodations used by college student survivors of traumatic brain injury. Brain Injury, 24(1), 13-26. https://doi.org/10. 3109/02699050903446823

Jacobs, K., Leopold, A., Hendricks, D. J., Sampson, E., Nardone, A., Lopez, K. B., Rumrill, P., Stauffer, C., Elias, E., Scherer, M., \& Dembe, J. (2017). Project Career: Perceived benefits of iPad apps among college students with traumatic brain injury (TBI). Work, 58, 45-50. https://doi.org/10.3233/WOR172596

Kennedy, M. R.T., Coelho, C., Turkstra, L., Ylvisaker, M., Moore Sohlberg, M., Yorkston, K., Chiou, H., \& Kan, P. (2008b). Intervention for executive functions after traumatic brain injury: A systematic review, meta-analysis and clinical recommendations. Neuropsychological Rehabilitation (18)3, 257-299. https://doi.org/10.1080/09602010701748644

Kennedy, M. R. T., Krause, M. O., \& Turkstra, L. S. (2008a). An electronic survey about college experiences after traumatic brain injury. Neurorehabilitation, 23, 511-520. https://doi. org/10.3233/NRE-2008-23607

Kennedy, M., \& Krause, M. (2011). Self-regulated learning in a dynamic coaching model for supporting college students with traumatic brain injury: Two case reports. Journal of Head Trauma Rehabilitation, 26(3), 212-223. https://doi. org/10.1097/HTR.0b013e318218dd0e

Kerr, Z. Y., Snook, E. M., Lynall, R. C., Dompier, T. P., Sales, L., Parsons, J. T. \& Hainline, B. (2015). Concussion-related protocols and preparticipation assessments used for incoming student-athletes in National Collegiate Athletic Association member institutions. Journal of Athletic Training, 50(11), 1174- 1181. https://doi.org/10.4085/1062-6050-50.11.11

Knollman-Porter, K., Constantinidou, F., \& Hutchinson Marron, K. (2014). Speech-language pathology and concussion management in intercollegiate athletics: The Miami University Concussion Management Program. American Journal of Speech-Language Pathology, 23, 507-519. https://doi. org/10.1044/2014_AJSLP-13-0126

Krause, M. O. P., \& Richards, S. R. (2014). Prevalence of traumatic brain injury and access to services in an undergraduate population: A pilot study. Brain Injury, 28(10), 1301-1310. https://doi.org/10.3109/02699052.2014.916416

LaForce Jr., R., \& Martin-MacLeod, L. (2001). Symptom cluster associated with mild traumatic brain injury in university students. Perceptual and Motor Skills, 93(1), 281-288. https://doi.org/10.2466/pms.2001.93.1.281

Ledbetter, A. K., Sohlberg, M. M., Fickas, S. F., Horney, M. A., \& McIntosh, K. (2019). Evaluation of a computer-based prompting intervention to improve essay writing in undergraduates with cognitive impairment after acquired brain injury, Neuropsychological Rehabilitation, 29(8), 1226-1255, https://doi.org/10.1080/09602011.2017.1383272

Leopold, A., Rumrill, P., Hendricks, D. J., Nardone, A., Sampson, E., Minton, D., Jacobs, K., Elias, E., \& Scherer, M. (2019). A mixed-methodological examination of participant experiences, activities, and outcomes in a technology and employment project for postsecondary students with traumatic 
brain injuries. Journal of Vocational Rehabilitation, 50, 3-11. https:/doi.org/10.3233/JVR-180983

Linquist, L. K., Love, H. C., \& Elbogen, E. B. (2017). Traumatic brain injury in Iraq and Afghanistan veterans: New results from a national random sample study. Journal of Neuropsychiatry and Clinical Neurosciences, 29(3), 254-259. https:// doi.org/10.1176/appi.neuropsych.16050100

MacLennan, D. L., \& MacLennan, D. C. (2008). Assessing readiness for post-secondary education after traumatic brain injury using a simulated college experience. NeuroRehabilitation, 23, 521-528. https://doi.org/10.3233/NRE-2008-23608

Madaus, J. W., Miller II, W. K., Vance, M. L. (2009). Veterans with disabilities in postsecondary education. Journal of Postsecondary Education and Disability, 22(1), 10-17.

Madaus, J. W. (2010). Let's be reasonable: Accommodations at the college level. In S. F. Shaw, J. W. Madaus, \& L. L. Dukes (Eds., pp. 37-63). Preparing students with disabilities for college: A practical guide for transition planning. Baltimore: Paul E. Brookes Publishing.

Martinez, S., \& Davalos, D. (2016). Investigating metacognition, cognition, and behavioral deficits of college students with acute traumatic brain injuries. Journal of American College Health, 64(5), 390-396. https://doi.org/10.1080/074 48481.2016.1167057

Mastropieri, M. A., Berkeley, S., McDuffie, K. A., Graff, H., Marshak, L., Conners, N. A., Diamond, C. M., Simpkins, P., Bowdey, F. R., Fulcher, A., Scruggs, T. E., \& Cuenca-Sanchez, Y. (2009). What is Published in the Field of Special Education? An Analysis of 11 Prominent Journals. Exceptional Children, 76(1), 95-109. https://doi.org/10.1177/001440290907600105

Mealings, M., Douglas, J., \& Olver, J. (2012). Considering the student perspective in returning to school after TBI: A literature review. Brain Injury, 26(10), 1165-1176. https://doi. org/10.3109/02699052.2012.672785

Nakase-Richardson, R., Sherer, M., Seel, R. T., Hart, T., Hanks, R., Arango-Lasprilla, J. C., Yablon, S. A., Sander, A. M., Barnett, S. D., Walker, W. C., \& Hammond, F. (2011). Utility of post-traumatic amnesia in predicting 1-year productivity following traumatic brain injury: Comparison of the Russell and Mississippi PTA classification intervals. Journal of Neurology, Neurosurgery \& Psychiatry, 82, 494-499. http:// dx.doi.org/10.1136/jnnp.2010.222489

Nardone, A., Sampson, E., Stauffer, C., Leopold, A., Jacobs, K., Hendricks, D. J., Elias, E., Chen, H., \& Rumrill, P. (2015). Project Career: A qualitative examination of five college students with traumatic brain injuries. NeuroRehabilitation, 37, 459-469. https://doi.org/10.3233/NRE-151274

NCAA Sport Science Institute. (2017). Concussion diagnosis and management best practices. Retrieved from http://www.ncaa. org/sport-science-institute/concussion-diagnosis-and-mana gement-best-practices

Newman, L., Wagner, M., Knokey, A.-M., Marder, C., Nagle, K., Shaver, D., Wei, X., with Cameto, R., Contreras, E., Ferguson, K., Greene, S., and Schwarting, M. (2011). The post-high school outcomes of young adults with disabilities up to 8 years after high school. A report from the National Longitudinal Transition Study-2 (NLTS2) (NCSER 2011-3005). Menlo Park, CA: SRI International. https://ies.ed. gov/ncser/pubs/20113005/pdf/20113005.pdf
Rumrill, P., Elias, E., Hendricks, D., Jacobs, K., Leopold, A., Nardone, A., Sampson, E., Scherer, M., Stauffer, C., \& McMahon, B. (2016). Promoting cognitive support technology use and employment success among postsecondary students with traumatic brain injury. Journal of Vocational Rehabilitation, 45, 53-61. https://doi.org/10.3233/JVR-160810

Scherer, M. J. (2005). Assessing the benefits of using assistive technologies and other supports for thinking, remembering and learning. Disability and Rehabilitation, 27(13), 731-739. https://doi.org/10.1080/09638280400014816

Shackelford, A. L. (2009). Documenting the needs of student veterans with disabilities: Intersection roadblocks, solutions, and legal realities. Journal of Postsecondary Education and Disability, 22(1), 36-42.

Sinski, J. B. (2012). Practice brief classroom strategies for teaching veterans with post-traumatic stress disorder and traumatic brain injury. Journal of Postsecondary Education and Disability, 25(1), 87-95.

Sohlberg, M. M., \& Ledbetter, A. K. (2016). Management of persistent cognitive symptoms after sport-related concussion. American Journal of Speech-Language Pathology, 25, 138149. https://doi.org/10.1044/2015_AJSLP-14-0128

State Vocational Rehabilitation Services Program, 85 F. R. 11848 (proposed Feb. 28, 2020) (to be codified at 34 CFR §361). https://www.govinfo.gov/content/pkg/FR-202002-28/pdf/2020-03208.pdf

Stewart-Scott, A. M., \& Douglas, J. M. (1998). Educational outcome for secondary and postsecondary students following traumatic brain injury. Brain Injury, 12(4), 317-331. https://doi.org/ 10.1080/026990598122629

Todis, B., \& Glang, A. (2008). Redefining success: Results of a qualitative study of postsecondary transition outcomes for youth with traumatic brain injury. Journal of Head Trauma Rehabilitation, 23(4), 252-263. https://doi.org/10.1097/01. HTR.0000327257.84622.bc

Todis, B., Glang, A., Bullis, M., Ettel, D., \& Hood, D. (2011). Longitudinal investigation of the post-high school transition experiences of adolescents with traumatic brain injury. Journal of Head Trauma Rehabilitation, 26(2), 138-149. https://doi.org/10.1097/HTR.0b013e3181e5a87a

United Nations. Department of Economic and Social Affairs. Disability. (2019). Disability Acts and Laws by Country/Area. https://www.un.org/development/desa/disabilities/disabilitylaws-and-acts-by-country-area.html

Weber, M., \& Edwards, M. G. (2010). The effect of brain injury terminology on university athletes' expected outcome from injury, familiarity and actual symptom report. Brain Injury, 24(11), 1364-1371. https://doi.org/10.3109/ 02699052.2010 .507110

Williamson, C. L., Norte, G. E., Broshek, D. K., Hart, J. M., \& Resch, J. E. (2018). Return to learn after sport-related concussion: A survey of secondary school and college athletic trainers. Journal of Athletic Training, 53(10), 990-1003. https://doi.org/10.4085/1062-6050-234-17

Zeng, W., Ju, S. J., \& Hord, C. (2018). A literature review of academic interventions for college students with learning disabilities. Learning Disability Quarterly, 41(3), 159-169. https://doi.org/10.1177/0731948718760999 